\title{
l'essor de la modélisation en centrifugeuse en géotechnique
}

\author{
the rise of geotechnical centrifuge modelling
}

\author{
J.-F. CORTÉ \\ Laboratoire Central des Ponts et Chaussées*
}

Rev. Franç. Géotech. n 48 , pp. 7-13 (juillet 1989)

\section{Résumé}

L'article donne en première partie un bref historique de l'évolution de la modélisation en centrifugeuse. II traite ensuite des apports des essais sur modèle réduit en centrifugeuse pour la simulation de problèmes réels, les études paramétriques, l'analyse des phénomènes mal connus et la validation des modèles numériques. Les caractéristiques des centrifugeuses françaises du CESTA et du LCPC sont présentées in fine.

\section{Abstract}

The first part of the paper gives a brief historical background of the development of centrifugal modelling. It then deals with the contributions of centrifuge model testing for the simulation of actual problems for parametric studies, for the investigation of misunderstood phenomena and for the validation of numerical models. The caracteristics of the French centrifuges of the CESTA and LCPC are described at the end. 


\section{INTRODUCTION}

Pour la plupart des problèmes géotechniques les efforts pondéraux ont en général une part essentielle dans les équilibres en jeu.

Les essais sur modèles réduits en centrifugeuse ont pour justification première de permettre de recréer au sein du corps d'épreuve, qui sera chaque fois que possible construit à partir des mêmes matériaux que le prototype, le même champ de forces de masse que dans l'ouvrage vraie grandeur. La règle de similitude qui en découle est simple et maintenant bien connue : l'intensité du champ de pesanteur créé par l'accélération centrifuge communiquée au modèle doit être inversement proportionnelle à l'échelle de réduction des dimensions de la maquette. Les facteurs d'échelles correspondant aux autres grandeurs physiques qui découlent de cette similitude ont été présentés à maintes reprises et ne sont pas réétablis ici; un tableau récapitulatif est donné par exemple dans l'article de SCOTT (1988).

Depuis une vingtaine d'années la modélisation en centrifugeuse est dans une phase de développement continuel, marqué par la multiplication des installations et des études relevant à la fois de la recherche et des applications industrielles. Il apparaissait ainsi opportun de présenter comment cette approche expérimentale s'est développée, dans quelles perspectives des études en centrifugeuse peuvent être entreprises et quels sont les moyens d'essais disponibles en France.

\subsection{Perspective historique}

L'idée d'effectuer des expériences sur modèle réduit avec une centrifugeuse semble avoir été présentée pour la première fois en 1869 par E. PHILLIPS, en France, à l'Académie des Sciences (PHILLIPS, 1869). Partant des équations différentielles d'équilibre des solides élastiques, il établit les relations de similitude qui devaient être safisfaites afin que l'ouvrage prototype et son modèle à échelle réduite présentent le même comportement. Lorsque la gravité est un facteur important, PHILLIPS suggéra d'utiliser la force centrifuge pour obtenir cette similitude.

C'est cependant au début des années 30 , que cette approche expérimentale allait être mise en œuvre, indépendamment, aux Etats-Unis et en Union Soviétique pour y être appliquée à différents problèmes.

Ainsi à PUniversité de Columbia, sur une toute petite centrifugeuse, BUCKI s'intéressa à l'apport des modèles réduits physiques pour l'étude de la stabilité des toits de mines, en combinant centrifugation et photoélasticité. D'autres travaux suivront dans le domaine minier et de la mécanique des roches, au Bureau des Mines de College Park et à l'Ecole des Mines de Missouri Rolla. Mais cette activité n'aura qu'un impact limité et ne s'accompagnera pas d'un développement des moyens expérimentaux. Aux Etats-Unis, la modélisation en centrifugeuse ne connaîtra de réel essor qu'à partir du milieu des années 70 , sous l'influence principale des résultats obtenus en Grande-Bretagne.

Tableau 1 - Grandes centrifugeuses utilisées pour les recherches géotechniques

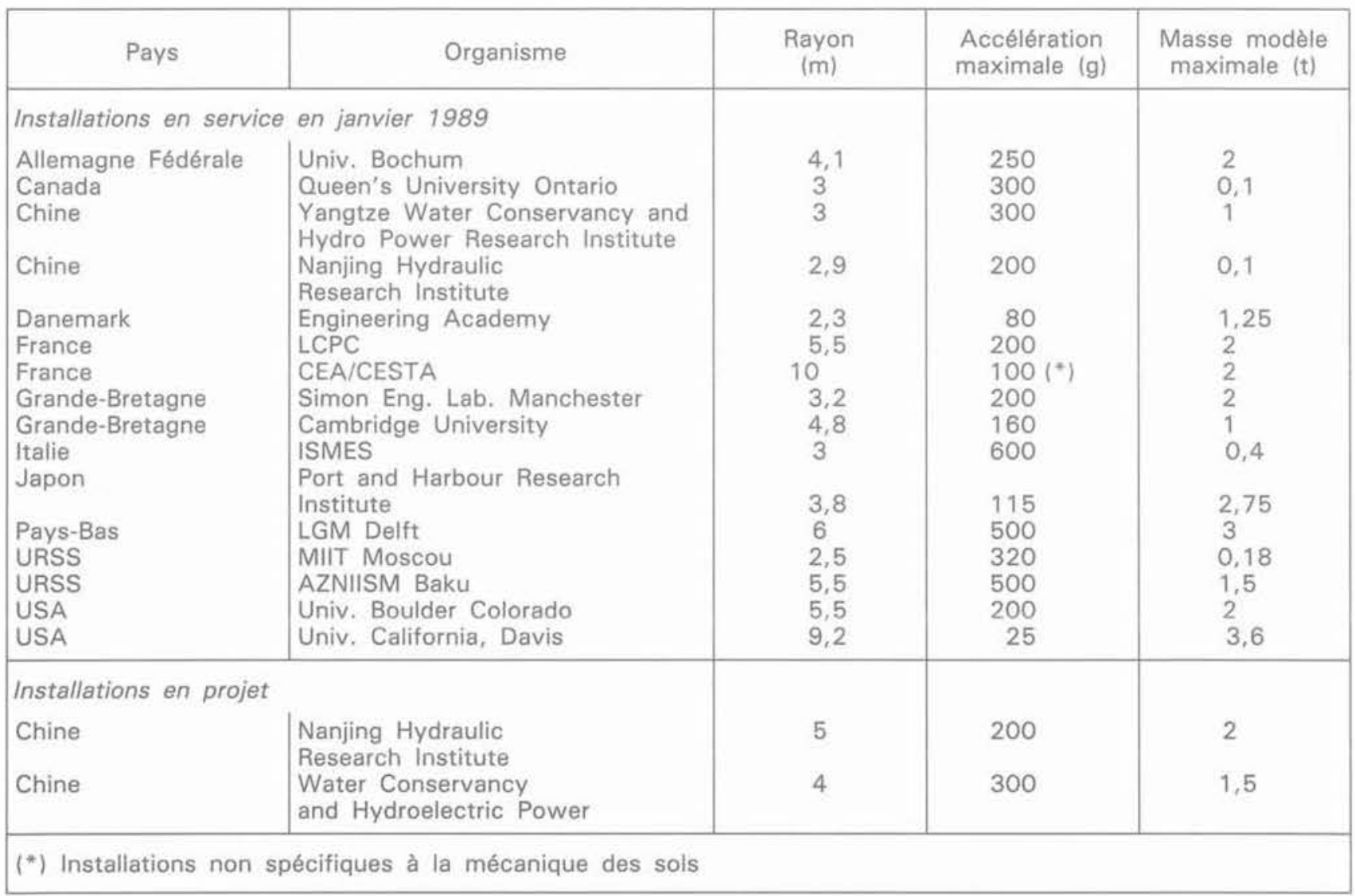


Si les premières expérimentations en centrifugeuse étaient entreprises à la même époque (1932) à Moscou au Laboratoire de Mécanique des Sols de l'Institut d'Hydrologie et d'Hydraulique Gidro-Texgeo, l'utilisation des centrifugeuses en mécanique des sols allait connaître un tout autre développement en Union Soviétique. La première application a été faite par POKROVSKI pour l'étude de la stabilité des berges du canal reliant les fleuves Usa et Volga. Rapidement, les résultats obtenus sur modèles réduits centrifugés furent utilisés pour le projet de barrages, remblais et digues. Au cours de la trentaine d'années qui suivit. on dénombre l'installation de plus de vingt centrifugeuses spécialisées pour les études géotechniques dans différents organismes de recherche.

En dépit d'une courte communication faite par POKROVSKI et FIODOROV dès le premier congrès de la Société Internationale de Mécanique des Sols en 1936, l'ensemble des travaux soviétiques, tant sur la théorie de la similitude en centrifugeuse que sur la conception de ces moyens d'essais et sur les expériences elles-mêmes, demeura ignoré du monde occidental. Leur révélation viendra tardivement, dans les années 60 , des traductions faites en Grande-Bretagne des ouvrages de SOKOLOVSKI, et de POKROVOSKI et FIODOROV.

Hors de l'Union Soviétique, ce sont les travaux menés au Japon et en Grande-Bretagne qui auront un impact majeur sur le développement de cette technique à travers le monde.

Au Japon, les premières expériences ont été faites par MIKASA en 1964 dans le but de valider la théorie qu'il venait d'établir sur la consolidation des sols fins en grandes déformations. L'équipe de l'Université d'Osaka poursuivit par l'étude de divers problèmes classiques de mécanique des sols (stabilité des pentes, capacité portante des fondations, tenue des murs de soutènement), mettant clairement en évidence l'importance d'une simulation correcte des forces de masse par rapport aux errements des essais sur modèle réduit, effectués classiquement en gravité normale. A cet égard les photographies publiées lors du Congrès International de Moscou en 1973 par MIKASA et TAKADA montrant les différences de champs de déplacements sous un poinçon en centrifugeuse et à $1 \mathrm{~g}$ demeurent une référence.

Depuis ces premiers travaux, un effort de recherche continu a été poursuivi au Japon avec la mise en place d'installations dans différents centres de recherche, y compris hors de la sphère universitaire comme à l'Institut de Recherche des Ports.

C'est en Grande-Bretagne, principalement sous l'impulsion de A.N. SCHOFIELD, que la modélisation en centrifugeuse s'est développée dans le monde occidental à partir de la fin des années 60. La découverte des travaux soviétiques allait en être l'élément initiateur, l'équipe de Cambridge trouvant dans cette méthode expérimentale le moyen de valider les concepts nouveaux d'x état critique ». En l'espace d'une vingtaine d'années, à Cambridge et à Manchester (à 1'Université et au Simon Engineering Laboratory), une multitude de problèmes différents et de complexité croissante vont être abordés : depuis les premières observations sur la tenue de tranchées et d'ouvrages en terre, jusqu'à la modélisation de la liquéfaction des sols sous l'action d'un séisme, en passant par des études paramétriques sur la stabilité de tunnels en terains meubles.

En France, les idées présentées par PHILLIPS seront mises en application pour les premières fois dans les années 70 par le Laboratoire de Mécanique des Solides de l'Ecole Polytechnique, puis par le Laboratoire Central des Ponts et Chaussées. Jusqu'en 1985, toutes les expérimentations ont eu lieu sur les installations du Commissariat à l'Energie Atomique, et principalement sur la machine du CESTA (CORTÉ, 1985)

Ces dix dernières années seront marquées par une forte croissance du nombre d'équipes qui se sont tournées vers la modélisation en centrifugeuse et par la construction de moyens d'essais de forte capacité conçus spécialement pour l'étude de problèmes de géotechniques (outre les pays déjà cités notons l'Allemagne Fédérale, le Canada, la Chine Populaire, le Danemark, l'Italie, les Pays-Bas, etc.).

Un état détaillé des connaissances par thème et par pays a été établi par le Comité Technique sur les centrifugeuses de la Société Internationale de Mécanique des Sols et des Travaux de Fondations. Le lecteur intéressé est invité à s'y reporter ; il y trouvera notamment une liste très complète des références bibliographiques sur les travaux en centrifugeuse publié jusqu'en 1985 (CRAIG et al., 1988). Les comptes rendus du Congrès Centrifugeuse 88 (CORTÉ, 1988) complètent ces références jusqu'à la période actuelle.

\section{PLACE ET APPORTS DES EXPÉRIENCES SUR MODÈLE RÉDUIT EN CENTRIFUGEUSE}

Si l'on considère l'ensemble des études faites sur modèle réduit en centrifugeuse, celles-ci peuvent être classées en quatre catégories selon l'objectif des expériences.

\subsection{La simulation d'un problème prototype réel précis}

Si cette application peut apparaitre comme a priori la plus évidente, rares sont encore cependant les situations pour lesquelles une situation réelle a été reproduite avec la fidélité nécessaire pour transposer directement les mesures faites sur le modèle réduit. La taille même des corps d'épreuve rend très difficile la reproduction de détails pouvant être déterminants pour le comportement de l'ouvrage réel (horizons faibles d'épaisseur centimétriques compromettant la stabilité des pentes ou d'ouvrages en terre de plusieurs dizaines de mètres de dimension caractéristique par exemple).

La simulation des phases de travaux demeure une opération difficile ce qui conduit à des différences entre les histoires de contraintes subies par le prototype et le modèle réduit. Les progrès très rapides en matière d'équipements et de robotisation des interventions sur modèle en cours de rotation permettent cependant d'envisager des simulations de plus en plus 
fidèles. Enfin les matériaux réels ne peuvent pas toujours être employés (taille des éléments, profils de consolidation en place...).

Le recours au modèle réduit physique pour l'étude d'un problème prototype spécifique trouve alors sa place dans l'analyse de certains aspects particuliers sur un corps d'épreuve dont la configuration est dérivée de la situation réelle simplifiée pour n'en conserver que les aspects significatifs. Il n'y a pas en fait au fond de différence fondamentale avec la démarche souvent non explicite retenue pour les études numériques qui admettent souvent des simplifications drastiques (emploi quasi systématique par exemple de modèles bidimensionnels, modèle en élasticité linéaire, etc.).

\subsection{Etudes paramétriques de problèmes simplifiés}

Les modèles réduits fournissent des corps d'épreuve beaucoup moins onéreux que les ouvrages réels, leurs caractéristiques tant géométriques que mécaniques et les conditions aux limites (souvent mal définissables dans la réalité), peuvent être mieux contrôlées par l'expérimentateur. On note ainsi un certain nombre d'applications faites à des problèmes de fondations notamment en vue de réévaluer diverses règles classiques concernant la capacité portante, règles qui ont jusqu'ici souvent été proposées sur la base d'essais sur modèle réduit à $1 \mathrm{~g}$, sans souci du respect de la similitude.

\subsection{L'étude des phénomènes mal connus}

La recherche de preuves expérimentales sur des phénomènes difficilement accessibles à l'observation sur site réel, a été le thème de prédilection des travaux menés en centrifugeuse. Nombreux sont encore les problèmes pour lesquels la capacité des méthodes de calcul s'avère insuffisante en raison de la complexité de la géométrie du problème posé (tupiquement effets tridimensionnels) ou de la loi de comportement des matériaux entrant en jeu.

La pertinence de la transposition des résultats obtenus aux situations réelles demande toujours une analyse critique qui n'est pas souvent menée avec rigueur. HABIB (1988) attire l'attention sur quelques limites importantes.

\subsection{Validation de modèles numériques}

L'évolution des outils de calcul pour le traitement des problèmes de milieux continus, est marquée par l'incorporation de modèles de complexité croissante pour la description des lois de comportement des géomatériaux. La valeur des résultats fournis par le calcul dépend non seulement de la qualité des dits modèles rhéologiques mais aussi de celle des algorithmes d'intégration; les problèmes ne sont pas à sous-estimer sur ce second point.

La « validation de modèles " n'a le plus souvent été effectuée que sur des problèmes homogènes, à travers la "prédiction " ou le recalcul d'essais de laboratoire sur échantillons. Les solutions analytiques de référence sont partiquement inexistantes hors quelques problèmes d'élasticité ou de plasticité parfaite.
Le recours à la prédiction de résultats d'expériences sur des problèmes aux limites rigoureusement contrôlés semble la seule voie. Dans cette optique, l'intérêt des essais en centrifugeuse tient à ce que des situations s'apparentant à des cas réels peuvent être reproduites en recréant en des points homologues des états de contrainte du même ordre de grandeur.

Les études sur modèle réduit en centrifugeuse ne constituent donc pas une approche venant en substitution des méthodes numériques, des essais en vraie grandeur ou des études rhéologiques, mais se placent comme un outil complémentaire. Il n'y a pas non plus une philosophie d'emploi unique, mais une analyse raisonnée à faire cas par cas.

\section{LES MOYENS DISPONIBLES EN FRANCE POUR LES ETUDES EN CENTRIFUGEUSES}

Avec les centrifugeuses du Centre d'Expérimentation Scientifique et Technique d'Aquitaine (CESTA) du CEA et du Laboratoire Central des Ponts et Chaussées de Nantes, la France dispose de deux moyens d'essais parmi les plus importants. Ces installations sont complémentaires de par leurs possibilités et les compétences qui ont été développées autour de ces installations.

\subsection{La centrifugeuse du CESTA}

Cette centrifugeuse a été construite en 1964 pour satisfaire les programmes développés par le CEA. Quelques années plus tard, elle a également été utilisée pour des travaux de diversification dans les domaines aéronautique et spatial et en mécanique des sols. C'est sur cette machine, qu'ont été effectuées en France jusqu'en 1984 l'ensemble des expériences en géotechnique.

La centrifugeuse (fig. 1), dans sa configuration pour les essais géotechniques, est équipée en extrémité de bras d'une nacelle pivotante. Les caractéristiques dimensionnelles principales sont :

- distance de l'axe aux articulations de la nacelle $8,40 \mathrm{~m}$;

- accélération maximale $100 \mathrm{~g}$;

- masse maximale utile du modèle $1000 \mathrm{~kg}$;

- plate-forme de la nacelle $1,2 \times 1,0 \mathrm{~m}$.

Pour les mesures, la machine dispose de 99 liaisons par contacts tournants, tandis que 16 voies servent à lalimentation des servitudes.

Différentes chaînes d'acquisition pilotées par ordinateur sont disponibles pour le suivi des divers paramètres mesurés.

L'une des spécificités de ce moyen d'essai est fournie par les équipements et les compétences en matière d'essais dynamiques. En collaboration avec le Laboratoire de Mécanique des Solides de l'Ecole Polytechnique, le CESTA a développé une méthode originale de simulation de séisme utilisant de petites charges explosives (ZELIKSON et al, 1981). La figure 2 montre le générateur d'onde sismique. 


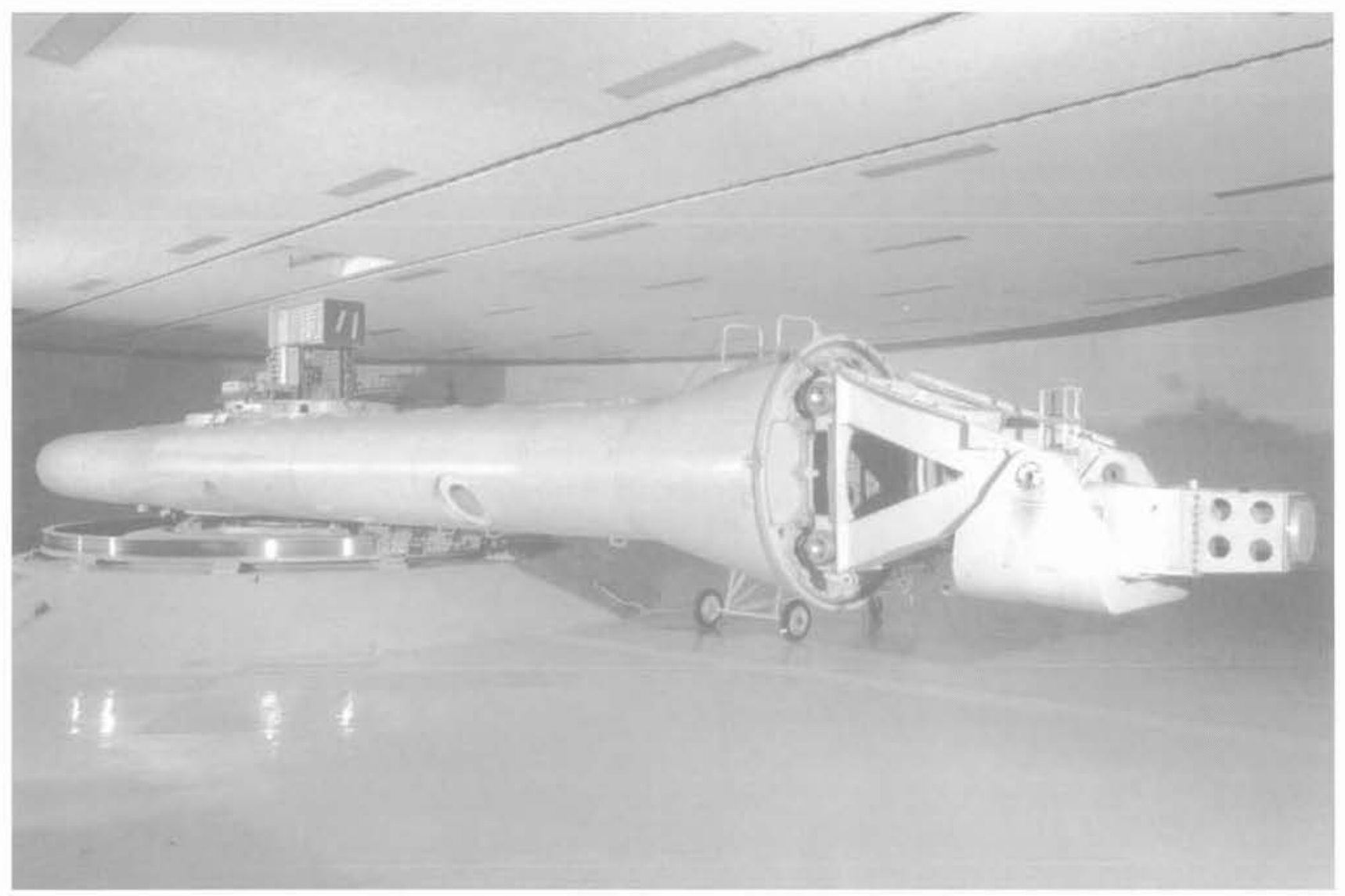

Fig. 1. - La centrifugeuse du CESTA.

Fig. 1. - The CESTA's centrifuge.

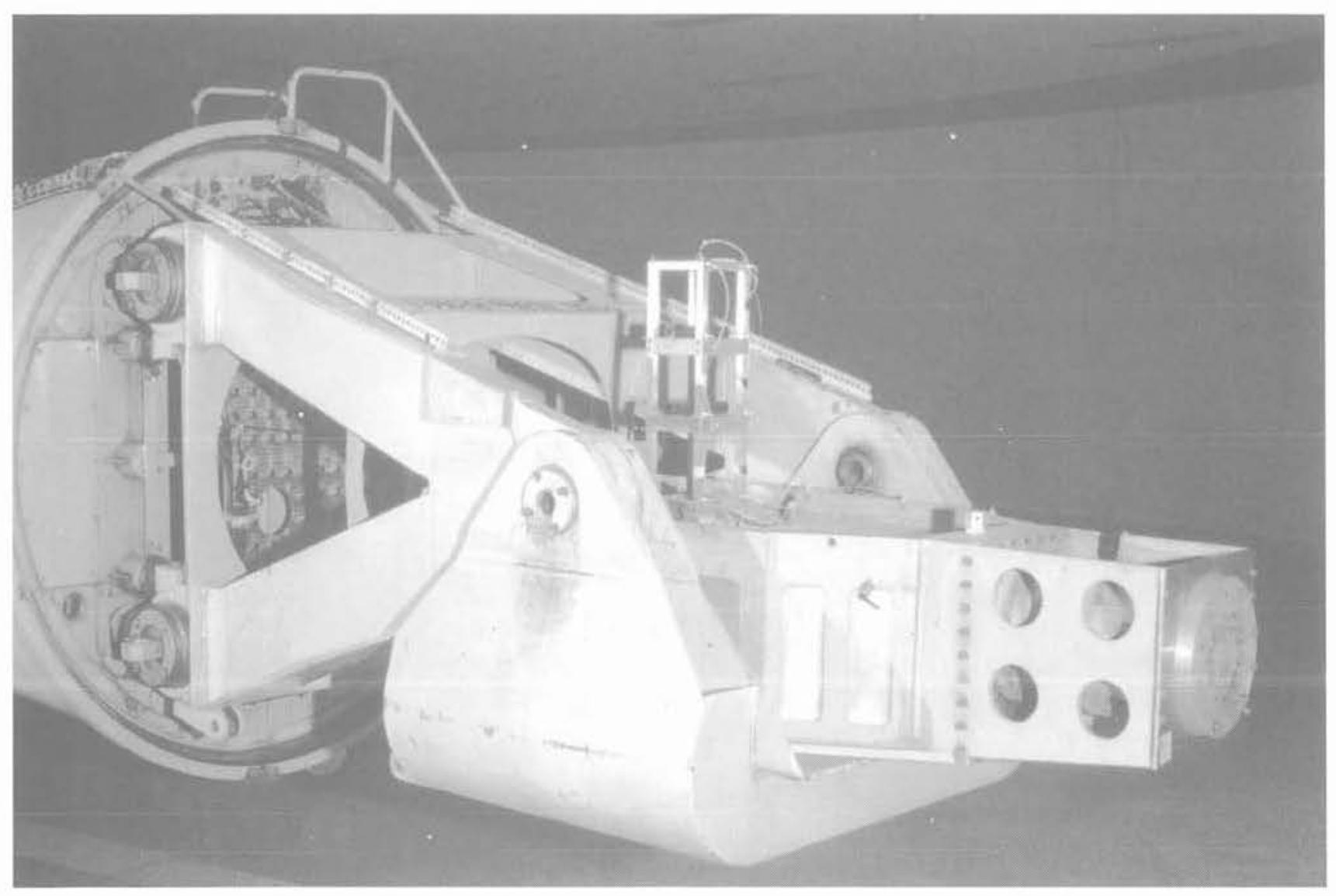

Fig. 2. - Générateur d'onde sismique pour essais en centrifugeuse (CESTA).

Fig. 2. - Seismic wave generator for centrifugal testing (CESTA). 
L'onde est générée par une série programmée de petites charges calibrées. L'interface entre la chambre d'explosion et le sol est constituée d'une membrane de caoutchouc qui communique le mouvement au sol de la maquette.

\subsection{La centrifugeuse du LCPC}

\section{Origine de ce moyen d'essai}

En 1973, le comité d'études préliminaires à l'action concertée * Les sciences de la terre et les problèmes d'aménagement, d'urbanisme et de construction s de la DGRST recommandait dans son rapport final l'installation d'une centrifugeuse pluriorganismes en France. Cette recommandation devait être confirmée par la Commission de concertation de la recherche du Comité Français de Mécanique des Sols en janvier 1974.

C'est seulement en 1980 que la DGRST donnait un avis positif pour l'inscription au budget 81 de la construction sur le site de Nantes du Laboratoire Central des Ponts et Chaussées, d'une grande centrifugeuse pour les recherches en mécanique des sols.

Cette installation est opérationnelle depuis décembre 1985 (fig. 3). Ce moyen d'essai national est mis en œuvre par le LCPC pour le programme d'études générales et de recherches du réseau des Laboratoires des Ponts et Chaussées, mais aussi pour le compte d'autres équipes de recherches et à des fins d'essais industriels.

\section{Caractéristiques essentielles}

Du point de vue de ses dimensions principales, la centrifugeuse du LCPC peut être caractérisée par les valeurs suivantes :

- rayon $5,50 \mathrm{~m}$ de l'axe à la plate-forme de la nacelle :

- accélération maximale $200 \mathrm{~g}$ (à $5 \mathrm{~m}$ de l'axe de rotation) :

- masse maximale du modèle $2000 \mathrm{~kg}$ à $100 \mathrm{~g}$; $500 \mathrm{~kg}$ à $200 \mathrm{~g}$ :

- * capacité * $200 \mathrm{t} \times \mathrm{g}$ (valeur maximale du produit masse embarquable $x$ accélération centrifuge applicable à cette masse).

Le domaine de la machine est précisé sur le diagramme de la figure 4.

Le détail des caractéristiques mécaniques et des aspects technologiques de ce moyen d'essais sont décrits par CORTÉ et al. (1986) et GARNIER et al. (1988) pour les aspects concernant l'instrumentation.

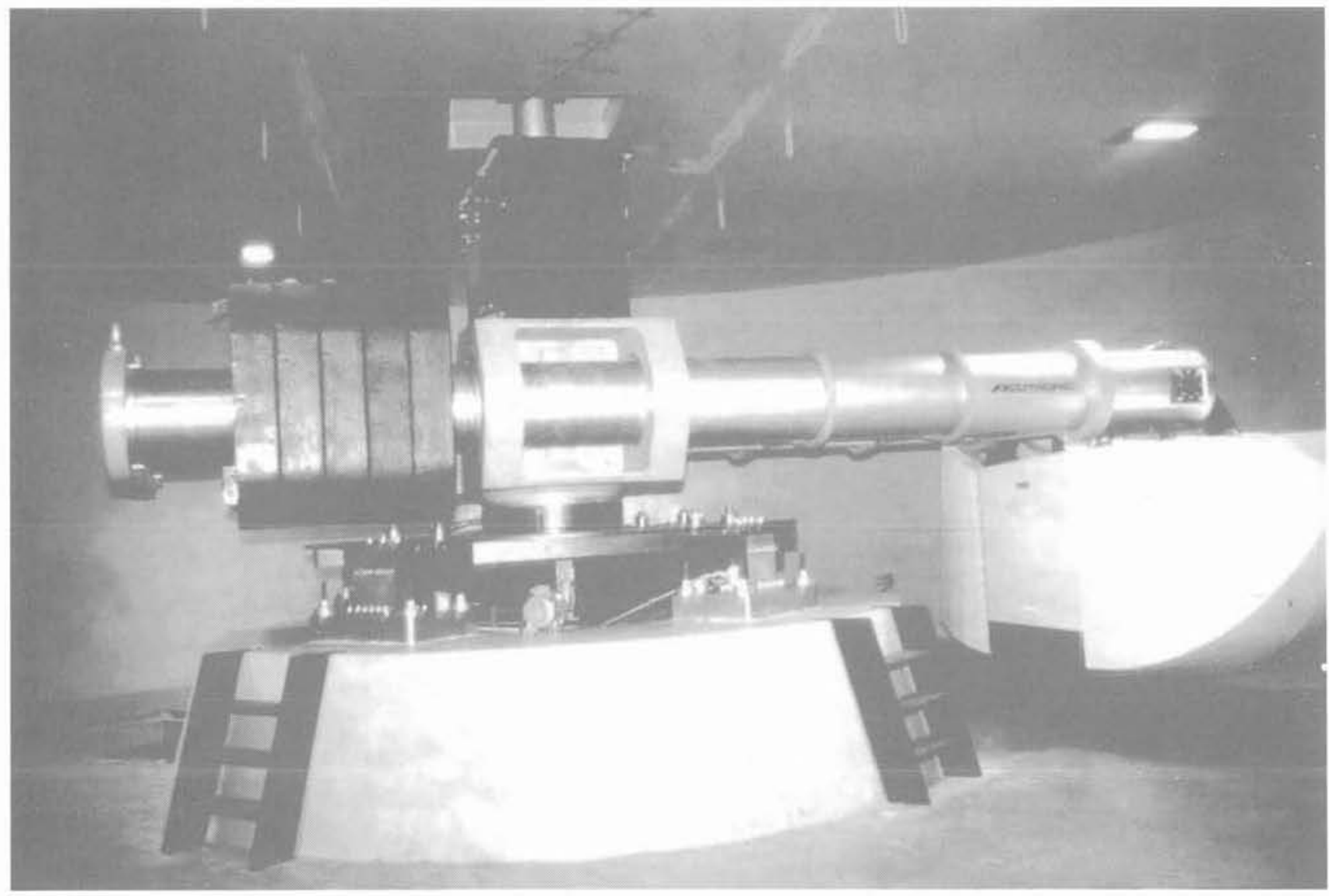

Fig. 3. - La centrifugeuse du LCPC.

Fig. 3 - The LCPC's centrifuge. 
(g) Accélération centrifuge

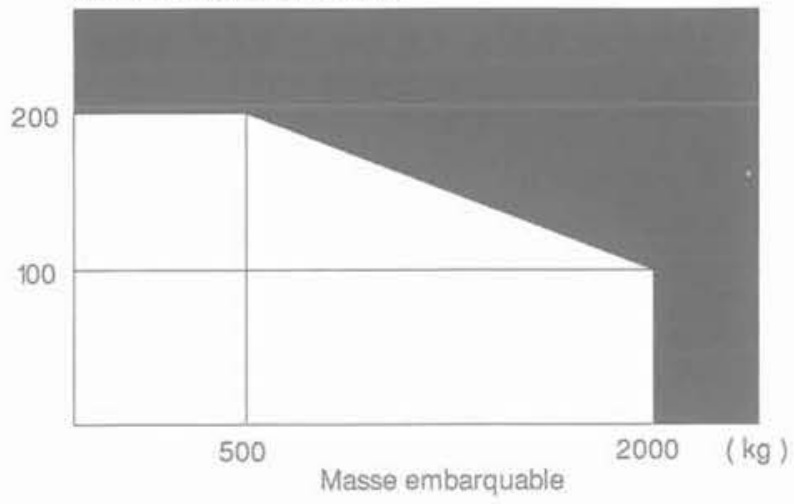

Fig. 4. - Domaine d'utilisation de la centrifugeuse du LCPC. Fig. 4. - Range of application of the LCPC's centrifuge.

La plate-forme de la nacelle offre une surface de 1.40 $\times 1,15 \mathrm{~m}$ permettant d'embarquer des grands modèles dont l'encombrement dépasse $2 \mathrm{~m}^{3}$. La structure très ouverte donne une grande accessibilité aux corps d'épreuve. Un ensemble de 101 contacts tournants mesures pour les signaux bas niveau, de 5 contacts tournants puissance et 5 joints tournants hydrauliques permettent d'établir en cours de rotation, tout type de liaison entre le modèle et le poste de commande :

- mesures prises sur le corps d'épreuve ;

- commande et alimentation d'équipements embarqués électriques, pneumatiques ou hydrauliques (moteurs, vérins, électro vannes...) ;

- apports fluides (air, eau, huile).

Une caractéristique originale importante est le fait que les chaînes d'acquisition des mesures soient embarquées sur le rotor et que les signaux soient numérisés avant passage par les contacts tournants. Ces dispositions permettent ainsi d'éliminer les problèmes de perturbation rencontrés avec l'acheminement des signaux analogiques à travers des contacts tournants et sur de grandes longueurs de lignes. L'utilisation du multiplexage permet en outre le suivi d'un nombre de paramètres très supérieur à celui fixé par le nombre de contacts tournants dans le cas de liaisons directes.

La liaison entre les chaînes embarquées et le calculateur du poste de pilotage est établie via un câble coaxial et 2 contacts tournants haute fréquence (liaison aux normes IEEE 428 sérialisée par 2 extenseurs HP-IB). Le débit atteint $50 \mathrm{ko} / \mathrm{s}$, un octet étant codé sur 22 bits.
Les caractéristiques des différentes chaînes de mesure et des liaisons sont décrites par GARNIER et al (1988).

Compte tenu de sa vocation géotechnique primitive, la centrifugeuse du LCPC a été conçue avec un ensemble de laboratoires et de matériels annexes pour la préparation des modèles de sol : trémie de pluviation automatisée pour les massifs de sable, bâtis de consolidation pour les sols fins. Pénétromètre et scissomètre sont également disponibles pour la détermination des caractéristiques mécaniques des sols en cours de rotation. Ces différents équipements sont aussi présentés dans l'article de GARNIER mentionné ci-dessus.

\section{BIBLIOGRAPHIE}

CORTÉ J.-F. (1985), Revue des essais par centrifu. gation en géotechnique en France de 1973 à 1985. Rapport des Laboratoires GT 5. Laboratoire Central des Ponts et Chaussées, Paris, $41 \mathrm{p}$.

CORTÉ J.F. (1988), Centrifuge'88. 610 pp. Balkema, Rotterdam.

CRAIG W.H., JAMES R.G., SCHOFIELD A.N. (1988), Centrifuges in soil mechanics, $266 \mathrm{pp}$. Balkema, Rotterdam.

GARNIER J., COTTINEAU L.M. (1988), La centrifugeuse du LCPC : moyens de préparation des modèles et instrumentation. Centrifuge' 88 , pp. 83-92, Balkema, Rotterdam.

HABIB P. (1988), Similitude et essais sur modèles réduits : validité de la modélisation. Revue Française de Géotechnique. Présent numéro.

MIKASA M., TAKADA N. (1973), Significance of centrifugal model test in soil mechanics. Proc. 8th Int. Conf. Soil Mechanics and Foundation Engineering, Vol. 1.2, pp. 273-278. Balkema, Rotterdam.

SCOTT R.F. (1988), Essais en centrifugeuse et technique de la modélisation. Revue Française de Géotechnique. Présent numéro.

ZELIKSON A., LEGUAY P., BADEL D. (1981), Représentation d'un séisme par des séquences de tirs d'explosifs en centrifugeuse. Proc. 6th Conf. Structural Mechanics in Reactor Technology, k4/11. 